\title{
Automatic CPAP titration with different self-setting devices in patients with obstructive sleep apnoea
}

\author{
A. Stammnitz, A. Jerrentrup, T. Penzel, J.H. Peter, C. Vogelmeier, H.F. Becker
}

\begin{abstract}
Automatic CPAP titration with different self-setting devices in patients with obstructive sleep apnoea. A. Stammnitz, A. Jerrentrup, T. Penzel, J.H. Peter, C. Vogelmeier, H.F. Becker. (C) ERS Journals Ltd 2004.
\end{abstract}

ABSTRACT: Autotitrating continuous positive airway pressure (CPAP) devices automatically adjust the pressure according to upper airway obstructions. The aim of this study was to compare the treatment effects of different automatic CPAP devices (AutoSet, Horizon and Virtuoso) with conventional CPAP in patients with obstructive sleep apnoea independently of financial manufacturer support.

Twelve male patients with obstructive sleep apnoea were submitted to a crossover study protocol with overnight polysomnography for 6 consecutive nights. After diagnostic polysomnography, the CPAP pressure was manually titrated. Over the next 4 nights, the patients were treated with any one of the three automatic CPAP devices or fixed CPAP in random order.

The apnoea/hypopnoea index on the diagnostic night was $67.3 \pm 21.7$ events $\cdot h^{-1}$, and was significantly reduced to $0.7 \pm 1.2,3.0 \pm 2.9,2.3 \pm 2.5$ and $12.0 \pm 13.6$ events $\cdot h^{-1}$ with the fixed CPAP, AutoSet, Horizon and Virtuoso devices respectively. An apnoeal hypopnoea index of $<5$ events $\cdot h^{-1}$, an indicator of optimal treatment, was achieved in all patients with fixed CPAP and in 10 patients using the Autoset and Horizon devices, but in only six of the 12 using the Virtuoso. The mean pressure was significantly lower with the AutoSet and Virtuoso devices, but not with the Horizon as compared to fixed CPAP. The maximum pressure was significantly higher with the Horizon.

It is concluded that automatic continuous positive airway pressure devices produce a significant reduction in apnoea/hypopnoea index; however, there is considerable difference in the efficacy of the various devices.

Eur Respir J 2004; 24: 273-278.
Dept of Respiratory Medicine \& Sleep Unit, Philipps University Marburg, Marburg an der Lahn, Germany.

\author{
Correspondence: H.F. Becker \\ University of Marburg \\ Dept of Respiratory Medicine \\ Sleep Unit \\ Baldingerstraße \\ 35033 Marburg \\ Germany \\ Fax: 4964212865405 \\ E-mail: HF.Becker@mailer.uni-marburg.de
}

Keywords: Automatic nasal continuous positive airway pressure titration

Received: June 302003

Accepted after revision: March 12004
Nasal continuous positive airway pressure (nCPAP) is the therapy of choice for patients with moderate-to-severe symptomatic sleep apnoea $[1,2]$ as it has been shown to improve quality of life [3] and cardiovascular risk factors such as arterial hypertension $[4,5]$ in controlled trials. The pressure applied during long-term treatment is generally determined by a technician in the sleep laboratory on the basis of a continuous polysomnographic recording. The treatment pressure is increased until apnoeas, hypopnoeas and snoring are prevented during all sleep stages and in the supine position. This fixed pressure is then used for home therapy. During the course of the night, the pressure that is needed to prevent upper airway obstruction varies according to the severity of upper airway obstruction, body position, sleep stage and other factors, such as nasal congestion and alcohol consumption [6-9]. Automatic CPAP devices were designed to increase the applied pressure when airway obstruction or snoring occurs and lower the pressure when the patient is awake or no obstruction occurs. The process of pressure titration might be simplified by these devices, thus saving time and costs.

The performance of automatic nCPAP devices can be evaluated by means of both bench studies and clinical studies. Bench studies testing the response of the device to numerous well-defined events under controlled conditions are essential during the developmental process before patients are treated. Clinical studies are then necessary to demonstrate that the device functions appropriately in clinical practice, where a variety factors may disturb the algorithm of the device.

The clinical studies published to date have tested the performance of a single device of the manufacturer sponsoring the study against no treatment [10-12], the pressure determination night [12-14] or fixed nCPAP $[12,15]$. The aim of the present study, therefore, was to compare the effectiveness of the three most frequently used automatic nCPAP devices in Germany (AutoSet ${ }^{\mathrm{TM}}$; ResMed, San Diego, CA, USA; Horizon; DeVilbiss Sunrise Medical, Inc., Carlsbad, CA, USA; and Virtuoso; Respironics, Inc., Murrysville, PA, USA) with fixed nCPAP using a crossover protocol independently of financial manufacturer support.

\section{Methods}

\section{Patients and protocol}

Over a period of 6 months, one patient per week could be studied. Each Tuesday, one patient with documented obstructive or mixed sleep apnoea (apnoea/hypopnoea index (AHI) of $>10$ events $\cdot h^{-1}$ ) in the diagnostic sleep study of the preceding night was asked to participate. If there was more than one eligible patient, patients were asked in alphabetical order. Thus, a group of consecutive unselected patients with 
moderate-to-severe obstructive and mixed sleep apnoea who commenced nCPAP treatment were studied. Exclusion criteria were predominantly central apnoeas, allergic rhinitis, upper airway infection, hypercapnic respiratory failure (arterial carbon dioxide tension of $>6.7 \mathrm{kPa}(>50 \mathrm{mmHg})$ ), heart failure (New York Heart Association Functional Class III-IV), claustrophobia or malignancy. Sixteen of the eligible patients consented to participate (one female and 15 males, aged $52 \pm 7.9$ yrs (mean $\pm \mathrm{SD}$ ), with a body mass index of $\left.32.3 \pm 4.0 \mathrm{~kg} \cdot \mathrm{m}^{-2}\right)$.

After the diagnostic sleep study, the patients received detailed information concerning nCPAP treatment, an appropriate nasal mask was chosen and the patients then tested fixed nCPAP at different pressure levels for $\sim 1 \mathrm{~h}$ during wakefulness until they felt comfortable breathing with the device.

All patients underwent manual CPAP titration during the second night. Over the following 4 polysomnographic study nights, the patients were treated in random order with one of three different self-adjusting CPAP devices or with fixed CPAP using the effective pressure that had been manually titrated. Four patients did not complete the study: three patients did not want to continue as they could not tolerate the unnecessarily high mask pressure during automatic CPAP (one patient each on the Virtuoso, Horizon and AutoSet devices) and one patient did not want to continue because he could not exhale with fixed nCPAP. The results of the 12 patients that completed the study are presented here.

The study protocol was approved by the ethics committee of Philipps University Marburg (Marburg an der Lahn, Germany), and all patients gave their written informed consent before inclusion in the study.

\section{Sleep studies}

The following parameters were recorded continuously using chart recorders (ED-16 and UD-8; Madaus Schwarzer, Munich, Germany): electroencephalography (2 leads (C3/A2 and C4/A1)), electro-oculography (2 leads), electromyography (chin), thoracic and abdominal movements by means of inductive plethysmography (Respitrace; Ambulatory Monitoring, Ardsley, NY, USA), oronasal airflow by means of thermistors, arterial oxygen saturation $\left(\mathrm{Sa}, \mathrm{O}_{2}\right)$ by means of finger oximetry (Nellcor, Hayward, CA, USA), and 1-channel electrocardiography. Snoring was detected with a surface microphone placed over the trachea.

CPAP therapy was introduced the night after the diagnostic study. All patients received CPAP via a nasal mask. The nasal mask was selected individually, and all patients used the same interface for all treatment nights. Instead of oronasal airflow, the pressure at the nasal mask was recorded using a piezoelectric pressure sensor (Sensortechnics, Puchheim, Germany) during the treatment nights.

\section{Continuous positive airway pressure titration}

Manual titration. The CPAP titration was started with the pressure set at $4 \mathrm{cmH}_{2} \mathrm{O}$. During sleep, the pressure was increased in steps of $1 \mathrm{cmH}_{2} \mathrm{O}$, until apnoeas, hypopnoeas and snoring were prevented during all sleep stages and in the supine position. This pressure was used for fixed nCPAP.

Automatic titration. Automatic titration was performed with the AutoSet, Horizon and Virtuoso devices. The AutoSet and Horizon utilise a pneumotachograph to detect flow limitation, snoring and apnoeas.
The Virtuoso device uses a pressure transducer to detect vibrations that occur together with apnoeas, hypopnoeas and snoring. In the present study, no upper or lower pressure limits were set.

\section{Data analysis}

The diagnostic night and the treatment nights were evaluated. Sleep stages were scored in 30-s epochs according to the criteria of RECHTSCHAFFEN and KALES [16]. An apnoea was defined as the cessation of oronasal airflow for $\geqslant 10 \mathrm{~s}$. A hypopnoea was defined as a reduction in oronasal airflow by $\geqslant 50 \%$ lasting for $\geqslant 10 \mathrm{~s}$, accompanied by a decrease in $\mathrm{Sa}, \mathrm{O}_{2}$ of $\geqslant 4 \%$ of the preceding stable $S \mathrm{a}, \mathrm{O}_{2}$. The number of apnoeas plus hypopnoeas per hour of sleep was calculated and reported as the AHI.

Arousal was defined according to American Sleep Disorders Association criteria [17]. The number of all electroencephalographic arousals per hour of sleep was defined as the total arousal index. The respiratory arousal index was defined as the number of respiratory disturbances (apnoeas, hypopnoeas and snoring episodes) accompanied by electroencephalographic arousal per hour of sleep. Nonrespiratory arousals were calculated as the difference between the total arousal index and the respiratory arousal index. The arousal-after-pressureelevation index was defined as the number of electroencephalographic arousals that occurred after a pressure elevation of the automatic CPAP devices per hour of sleep.

$\mathrm{Sa}, \mathrm{O}_{2}$ was recorded continuously and minimal, maximal and mean $\mathrm{Sa}, \mathrm{O}_{2}$, as well as the percentage of total sleep time (TST) spent at an $\mathrm{Sa}, \mathrm{O}_{2}$ of $<90 \%$, were calculated.

Mask pressure was continuously recorded and minimal, maximal and mean mask pressure, as well as the percentage of TST spent with a mask pressure greater than that of fixed CPAP, were calculated.

The evaluation was performed by an experienced technician otherwise not involved in the study.

\section{Statistical analysis}

Data were computed as mean \pm SD for descriptive purposes. The different treatment modalities were compared using Friedman's test. Pairwise comparisons were carried out by means of the Wilcoxon test. Significance was assumed at a p-value of $<0.05$.

\section{Results}

\section{Respiratory disturbances}

Mean AHI was significantly decreased with fixed CPAP and all of the automatic CPAP devices (fig. 1). The AHI with fixed CPAP was significantly lower than with all of the automatic nCPAP devices. The AHI with the AutoSet and Horizon devices was significantly lower than with the Virtuoso. An AHI of $<5$ events $\cdot h^{-1}$, an indicator of optimal treatment, was seen in all patients using fixed CPAP and in 10 of the 12 patients with the Horizon and AutoSet devices, but in only six of the 12 patients with the Virtuoso.

\section{Sleep structure}

Table 1 shows the TST and sleep stage distribution of the diagnostic and treatment nights. The TST was not 


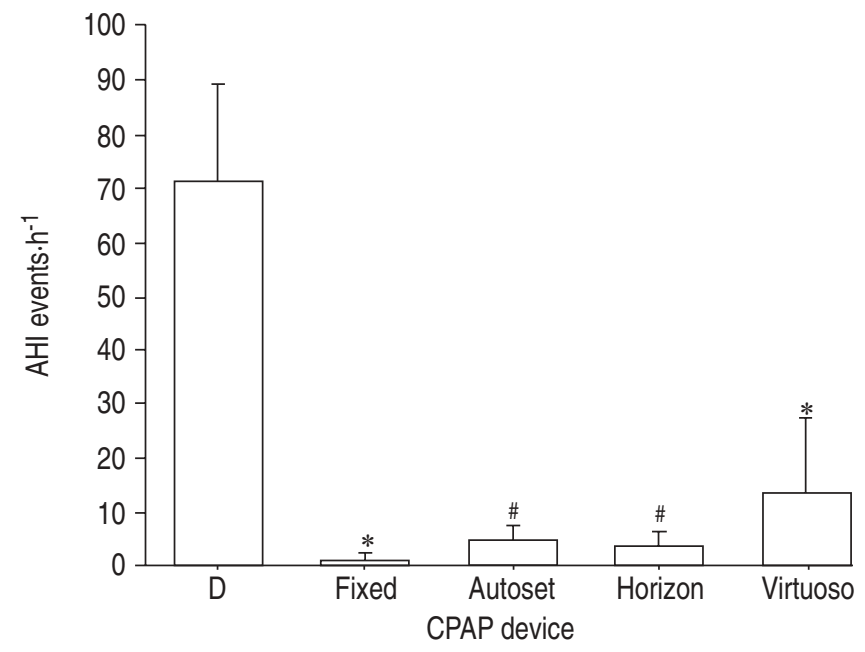

Fig. 1.-Apnoea/hypopnoea index (AHI) during the diagnostic (D) and treatment nights using various continuous positive airway pressure (CPAP) devices. Data are presented as mean \pm SD. *: $\mathrm{p}<0.05$ versus all other groups; ${ }^{\#}: \mathrm{p}<0.05$ versus diagnostic night, fixed CPAP and Virtuoso.

significantly different on any of the 5 study nights. The stage 3-4 nonrapid eye movement and rapid eye movement sleep stages increased on all 4 treatment nights compared to the diagnostic night. No significant differences concerning sleep stages were found between fixed CPAP and the automatic CPAP devices.

\section{Arousal}

Figure 2 shows the individual results for total arousal index. No significant differences were found between the automatic CPAP devices compared to fixed CPAP. Figure 3 gives an overview of the arousals separated into respiratory and nonrespiratory arousals, as well as arousal after pressure elevation. The number of respiratory arousals with fixed CPAP was significantly lower than with the AutoSet and Virtuoso devices. No significant difference was seen between the AutoSet and the Horizon. With the Virtuoso, the number of respiratory arousals was significantly higher than with the Horizon and fixed CPAP.

The arousals seen after an increase in pressure by automatic CPAP devices ranged $0.8-1.3$ events $\cdot h^{-1}$ and were not significantly different between the automatic CPAP devices. In addition, no significant difference was found in the number of nonrespiratory arousals between the 4 treatment nights.

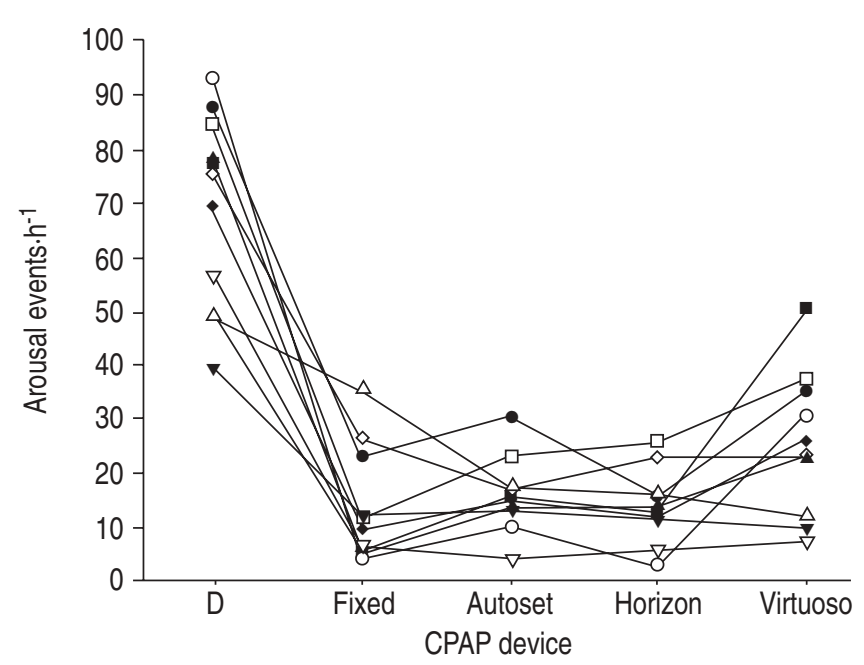

Fig. 2. - Individual results for total arousal index during the diagnostic (D) and treatment nights using various continuous positive airway pressure (CPAP) devices.

\section{Oxygen saturation}

The minimal and mean $\mathrm{Sa}, \mathrm{O}_{2}$ during the treatment nights are shown in table 2 . The differences found in minimal and mean $\mathrm{Sa}, \mathrm{O}_{2}$ and the percentage of TST spent with an $\mathrm{Sa}_{2} \mathrm{O}_{2}$ of $<90 \%$ did not differ significantly between automatic CPAP devices compared to fixed CPAP.

\section{Mask pressure}

Figure 4 shows the mean mask pressure with fixed CPAP, as well as the mean and maximal pressures with the automatic CPAP devices. The mean pressure with fixed CPAP was $9.9 \pm 1.8 \mathrm{cmH}_{2} \mathrm{O}$, and was significantly lower with the AutoSet $\left(7.3 \pm 1.6 \mathrm{cmH}_{2} \mathrm{O}\right)$ and Virtuoso $\left(6.5 \pm 2.3 \mathrm{cmH}_{2} \mathrm{O}\right)$ devices. No significant difference was found for the Horizon $\left(8.5 \pm 2.8 \mathrm{cmH}_{2} \mathrm{O}\right)$ compared to fixed CPAP. The mean pressures did not differ significantly between the three automatic CPAP devices.

Figure 4 also shows that, with automatic CPAP devices, higher maximal pressures were applied compared to fixed CPAP $\left(11.4 \pm 1.9,13.3 \pm 3.5\right.$ and $10.5 \pm 3.3 \mathrm{cmH}_{2} \mathrm{O}$ for the AutoSet, Horizon and Virtuoso devices, respectively). Compared to fixed CPAP, this difference was not significant for the AutoSet and Virtuoso, but maximum pressure with the Horizon was significantly higher than with fixed CPAP and the AutoSet and Virtuoso. The percentage of TST spent with a pressure higher than with fixed CPAP did not differ significantly between the automatic CPAP devices (table 3).

Table 1. - Total sleep time (TST) and sleep stages of the diagnostic and treatment nights

\begin{tabular}{lcccc}
\hline & Diagnostic night & Fixed CPAP & \multicolumn{2}{c}{ Automatic CPAP } \\
\cline { 3 - 5 } & & AutoSet & Horizon \\
\hline TST min & $363.7 \pm 61.4$ & $374.6 \pm 86.5$ & $339.2 \pm 66.7$ & $356.5 \pm 52.6$ \\
NREM \% & $14.9 \pm 12.0$ & & & $339.5 \pm 51.3$ \\
Stage 1 & $70.2 \pm 11.8$ & $52.3 \pm 7.5^{*}$ & $9.7 \pm 6.2^{*}$ & $9.1 \pm 6.6^{*}$ \\
Stage 2 & $5.8 \pm 6.5$ & $18.3 \pm 5.7^{*}$ & $51.9 \pm 10.0^{*}$ & $54.2 \pm 7.2^{*}$ \\
Stage 3-4 & $9.0 \pm 3.8$ & $21.4 \pm 6.1^{*}$ & $16.8 \pm 6.1^{*}$ & $11.2 \pm 5.4$ \\
REM \% & & $21.6 \pm 9.0^{*}$ & $50.2 \pm 9.8^{*}$ \\
\hline
\end{tabular}

Data are presented as mean \pm SD. CPAP: continuous positive airway pressure; NREM: nonrapid eye movement; REM: rapid eye movement. $*: \mathrm{p}<0.05$ versus diagnostic night. 


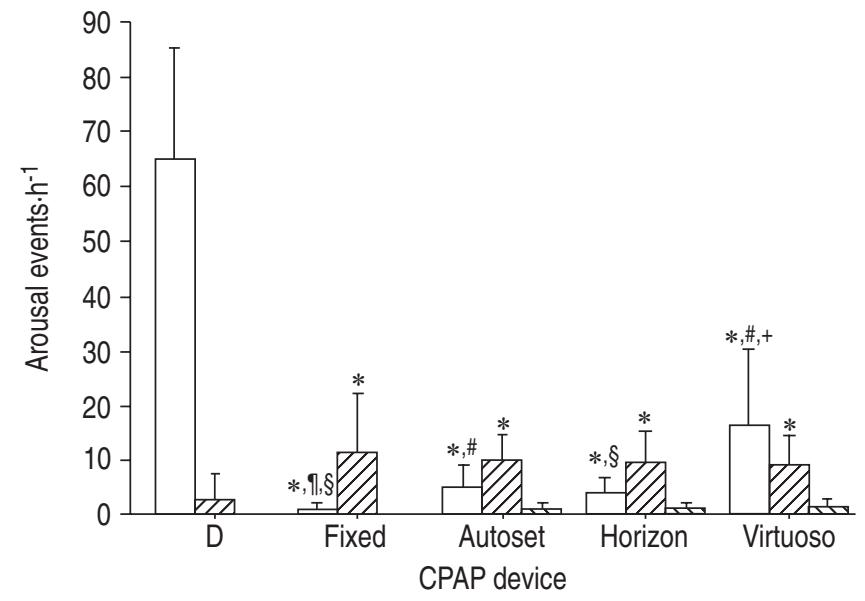

Fig. 3.-Arousal caused by respiratory events ( $\square$; apnoeas, hypopnoeas and snoring), as well as nonrespiratory events $(\mathbb{Z})$ or pressure elevation $(\mathbb{\mathbb { Q }})$, during the diagnostic (D) and treatment nights using various continuous positive airway pressure (CPAP) devices. Data are presented as mean \pm SD. *: $\mathrm{p}<0.05$ versus diagnostic night; ${ }^{\#}: \mathrm{p}<0.05$ versus fixed CPAP; ${ }^{\text {ๆ: }} \mathrm{p}<0.05$ versus AutoSet; ${ }^{+}: \mathrm{p}<0.05$ versus Horizon; ${ }^{\S}: \mathrm{p}<0.05$ versus Virtuoso.

\section{Discussion}

The automatic CPAP devices tested in the present study significantly reduced the AHI of patients with moderate-tosevere obstructive sleep apnoea. Compared to fixed CPAP, the AHI was significantly higher with all automatic nCPAP devices. Optimal treatment, with an AHI of $<5$ events $\cdot \mathrm{h}^{-1}$, generally accepted as normal [18], was seen in all patients by use of fixed CPAP and in 10 of the 12 patients with the AutoSet and Horizon devices, but in only six of the 12 patients with the Virtuoso. With the automatic CPAP devices, $S \mathrm{a}, \mathrm{O}_{2}$, arousal index and sleep structure were substantially improved in a way that was similar to that with fixed CPAP. The mean CPAP pressure of the automatic CPAP devices was 1.4-3.5 $\mathrm{cmH}_{2} \mathrm{O}$ lower than with fixed CPAP, which was significant for the AutoSet and Virtuoso. However, with all autotitrating CPAP devices, the maximum pressure applied throughout the night was $0.6-3.4 \mathrm{cmH}_{2} \mathrm{O}$ higher than with fixed CPAP.

A prospective controlled randomised crossover design was chosen for the present study. All patients were studied polysomnographically over six consecutive nights. Although all of the patients underwent a diagnostic study on the first night and manual titration of fixed CPAP on the following night, the order in which the three automatic CPAP devices and fixed CPAP were applied over the subsequent 4 nights was randomised and balanced. Thus any sequence effect was avoided. In contrast to many previous studies, the effect of automatic CPAP devices was compared with effective fixed CPAP, whereas several previous studies compared automatic CPAP treatment alone with the diagnostic night [19] or with

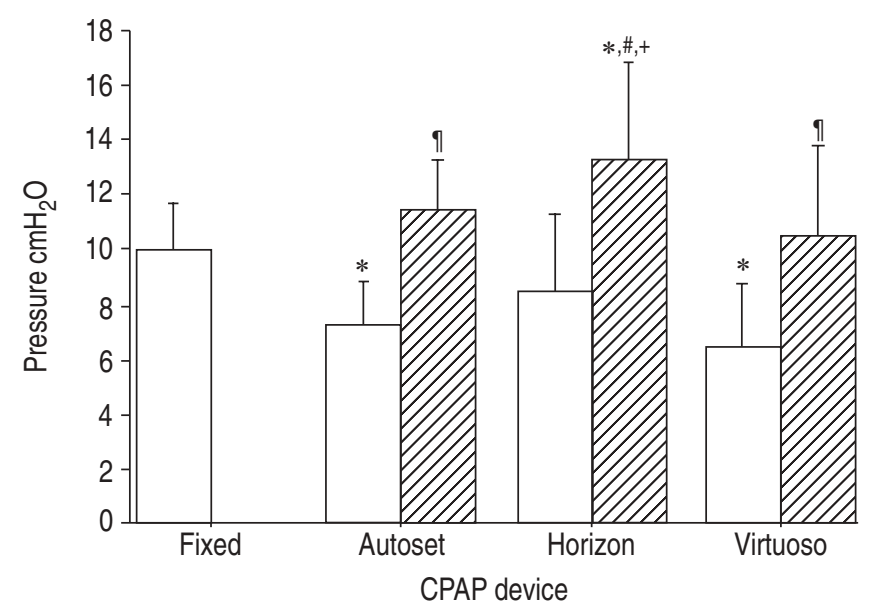

Fig. 4.-Mean $(\square)$ and maximum $(\mathbb{Z})$ pressure with the various continuous positive airway pressure (CPAP) devices. Data are presented as mean \pm SD. *: $\mathrm{p}<0.05$ versus fixed CPAP; ${ }^{\#}: \mathrm{p}<0.05$ versus AutoSet; ${ }^{\uparrow}: \mathrm{p}<0.05$ versus Horizon; ${ }^{+}: \mathrm{p}<0.05$ versus Virtuoso.

the results of the pressure determination night for fixed nCPAP $[13,20]$.

The present study confirms the significant reduction in AHI obtained with individual automatic CPAP devices [11, 14, 15, 21-26]. In addition and for the first time, it provides comparative data demonstrating substantial differences in the effectiveness of different automatic CPAP devices and fixed nCPAP.

The greatest AHI reduction of $>98 \%$ was achieved with fixed CPAP. In two previous studies, the results with automatic CPAP were better than those with fixed CPAP $[12,13]$, most probably due to the fact that, unlike in the present study, no attempt was made by these authors to eliminate snoring as a symptom of persisting partial upper airway obstruction. A further cause of the relatively poor results with fixed CPAP in these studies may be that the AHI of the manual titration night, with longer periods of ineffective fixed nCPAP, was compared with the results of automatic CPAP. SCHARF et al. [27], using a study design similar to that in the present study, also found a lower AHI with fixed CPAP compared to automatic CPAP (the Horizon), although the results of the two treatment modalities did not differ significantly.

Although all three automatic devices studied significantly reduced the AHI, substantial differences in effectiveness were

Table 3.-Time spent with a pressure $(P)$ higher than with fixed continuous positive airway pressure (CPAP)

\begin{tabular}{lccc}
\hline & AutoSet & Horizon & Virtuoso \\
\hline TST at $P>$ fixed CPAP $\%$ & $22.8 \pm 33.8$ & $38.6 \pm 32.9$ & $6.1 \pm 12.3$ \\
\hline
\end{tabular}

Data are presented as mean \pm SD. TST: total sleep time.

Table 2. - Arterial oxygen saturation $\left(\mathrm{Sa}, \mathrm{O}_{2}\right)$ and time spent at an $\mathrm{Sa}, \mathrm{O}_{2}$ of $<90 \%$ during treatment nights

\begin{tabular}{lllll}
\hline & Fixed CPAP & \multicolumn{3}{c}{ Automatic CPAP } \\
\cline { 3 - 5 } & & AutoSet & Horizon & Virtuoso \\
\hline Mean $\mathrm{Sa}_{2} \mathrm{O}_{2} \%$ & $95.2 \pm 1.6$ & $95.8 \pm 1.3$ & $95.5 \pm 1.0$ & $94.7 \pm 3.1$ \\
Minimum $S \mathrm{a}, \mathrm{O}_{2} \%$ & $84.9 \pm 3.6$ & $84.5 \pm 5.1$ & $86.4 \pm 2.6$ & $81.5 \pm 6.5$ \\
TST at $<90 \% \mathrm{Sa}, \mathrm{O}_{2} \%$ & $0.59 \pm 0.74$ & $0.98 \pm 1.70$ & $0.33 \pm 0.36$ & $9.29 \pm 26.63$ \\
\hline
\end{tabular}

Data are presented as mean $\pm \mathrm{SD}$. CPAP: continuous positive airway pressure; TST: total sleep time. 
shown. The AutoSet as well as the Horizon device reduced the AHI by $>90 \%$ and thus came close to the optimal result achieved with fixed nCPAP. Although the AHI with both devices was significantly higher than with fixed CPAP, these differences were small and most probably clinically irrelevant. With the Virtuoso, mean AHI was significantly reduced by $>80 \%$ compared to the diagnostic night; however, the AHI remained at $>10$ events $\cdot h^{-1}$ in six of the 12 patients studied and mean AHI with the Virtuoso was substantially higher than with fixed CPAP. Since the Virtuoso does not utilise a pneumotachograph, it does not reliably detect the obstructive apnoeas with complete upper airway occlusion that occur suddenly without previous snoring in some patients. Conversely, the system reacts adequately with an increase in pressure to partial airway obstruction such as obstructive hypopnoeas and snoring. SHARMA et al. [20] demonstrated a reduction in AHI from 50.8 to 6.1 events $\cdot \mathrm{h}^{-1}$ with the Virtuoso, but to $\sim 3$ events $\cdot h^{-1}$ with fixed CPAP. As in the present study, it was observed that the Virtuoso did not increase pressure despite upper airway obstruction in seven of 20 patients.

Normalisation of sleep stages was observed during the treatment nights. The longest duration of slow-wave sleep was found with fixed CPAP, most probably caused by the largest reduction in AHI and respiratory arousal index with this device.

Compared to the other three devices, treatment with the Virtuoso led to a substantially higher total arousal index, which can be attributed to persisting breathing disturbances. As expected, the number of nonrespiratory arousals did not differ significantly between fixed CPAP and any of the three automatic nCPAP machines in the present study, and they even occurred less frequently than in a study in healthy subjects [28]. Arousal after pressure elevation during automatic CPAP accounted for $<7 \%$ of all arousals. Thus, arousals caused by pressure increase is not a relevant problem in the devices tested.

The mean treatment pressure of all of the automatic CPAP devices was lower than that used for fixed CPAP. With the Autoset and Horizon, the mean pressure was lower $(27 \%$ and $14 \%$, respectively) than with fixed CPAP. Treatment with these two devices was slightly less effective than with fixed CPAP. The lowest mean mask pressure was applied by the Virtuoso (34\% lower). However, the Virtuoso failed to increase pressure in reaction to breathing disorders, leading to the highest $\mathrm{AHI}$ and arousals and the lowest $\mathrm{Sa}, \mathrm{O}_{2}$ with treatment.

The maximum pressure applied during automatic CPAP treatment clearly exceeded the pressure used for fixed CPAP, and patients spent up to $38 \%$ (the Horizon) of TST with a pressure that was higher than with fixed CPAP; thus, unnecessarily high mask pressure may be applied by autotitrating devices.

Based on the present study, it cannot be concluded that automatic CPAP is as effective in an unattended situation in the patient's home. Several observations were made of the automatic CPAP machines erroneously increasing pressure due to mask leaks, and, in three patients, this was the reason for withdrawal of their consent to continue the study. Therefore, unattended automatic nCPAP without an upper pressure limit may deter patients from nCPAP therapy. Furthermore, the only aspect of the initiation of CPAP therapy that was dealt with by the automatic device was pressure determination. All of the other aspects, such as mask adaptation, prevention or removal of leak, and patient support, were performed by experienced technicians.

The present study demonstrates substantial differences in the performance of automatic nCPAP devices in the clinical setting. In addition to bench testing, clinical testing of the different devices, as performed here, is essential for the evaluation of the performance and efficacy of automatic CPAP in routine use. Devices that do not achieve a reduction in breathing disorders similar to those obtained with fixed nCPAP should not be used. From the present study study, the current authors have come to the conclusion that fixed nCPAP should be used in the first line of treatment. If patients report discomfort with fixed nCPAP, automatic CPAP with devices that lead to a prevention of breathing disorders comparable to those obtained with fixed nCPAP may be a valuable additional treatment option.

It is concluded that two of the three automatic continuous positive airway pressure devices tested effectively treat patients with uncomplicated obstructive sleep apnoea in the sleep laboratory and provide treatment results that are comparable to those obtained with fixed continuous positive airway pressure, the standard treatment at present. The present study also demonstrates that not all autotitrating devices are equally effective. The Virtuoso device did not work properly in $50 \%$ of the patients, and, as a consequence, is no longer commercially available in Germany.

\begin{abstract}
Acknowledgements. The authors would like to thank W. Althaus for the sleep study scoring and U. Brandenburg for technical assistance. W. Cassel provided valuable assistance with the statistical analysis.
\end{abstract}

\section{References}

1. Sullivan C, Issa F, Berthon Jones M, Eves L. Reversal of obstructive sleep apnoea by continuous positive airway pressure applied through the nares. Lancet 1981; 1: 862-865.

2. Anon. American Thoracic Society Official Statement. Indications and standards for use of nasal continuous positive airway pressure (CPAP) in sleep apnea syndrome. Am J Respir Crit Care Med 1994; 50: 1738-1745.

3. Jenkinson C, Davies RJ, Mullins R, Stradling JR. Comparison of therapeutic and subtherapeutic nasal continuous positive airway pressure for obstructive sleep apnoea: a randomised prospective parallel trial. Lancet 1999; 353: 2100-2105.

4. Pepperell JC, Ramdassingh-Dow S, Crosthwaite N, et al. Ambulatory blood pressure after therapeutic and subtherapeutic nasal continuous positive airway pressure for obstructive sleep apnoea: a randomised parallel trial. Lancet 2002; 360: 204-210.

5. Becker HF, Jerrentrup A, Ploch $\mathrm{T}$, et al. Effect of nasal continuous positive airway pressure treatment on blood pressure in patients with obstructive sleep apnea. Circulation 2003; 107: 68-73.

6. Deegan PC, McNicholas WT. Pathophysiology of obstructive sleep apnoea. Eur Respir J 1995; 8: 1161-1178.

7. Neill AM, Angus SM, Sajkov D, McEvoy RD. Effects of sleep posture on upper airway stability in patients with obstructive sleep apnea. Am J Respir Crit Care Med 1997; 155: 199-204.

8. Oksenberg A, Silverberg DS, Arons E, Radwan H. The sleep supine position has a major effect on optimal nasal continuous positive airway pressure: relationship with rapid eye movements and non-rapid eye movements sleep, body mass index, respiratory disturbance index, and age. Chest 1999; 116: 1000-1006.

9. Teschler H, Berthon-Jones M, Wessendorf T, Meyer HJ, Konietzko N. Influence of moderate alcohol consumption on obstructive sleep apnoea with and without AutoSet nasal CPAP therapy. Eur Respir J 1996; 9: 2371-2377.

10. Pepin J, Nadjafizadeh H, Benkheira N, Levy P. A selfadjusted CPAP device for OSA using snoring detection and 
pressure changes: clinical validity. Am J Respir Crit Care Med 1995; A534.

11. Lofaso F, Lorino A, Duizabo D, et al. Evaluation of an auto-nCPAP device based on snoring detection. Eur Respir J 1996; 9: 1795-1800.

12. Teschler H, Berthon Jones M, Thompson A, Henkel A, Henry J, Konietzko N. Automated continuous positive airway pressure titration for obstructive sleep apnea syndrome. Am J Respir Crit Care Med 1996; 154: 734-740.

13. Lloberes P, Ballester E, Montserrat JM, et al. Comparison of manual and automatic CPAP titration in patients with sleep apnea/hypopnea syndrome. Am J Respir Crit Care Med 1996; 154: $1755-1758$.

14. Stradling J, Barbour C, Pitson D, Davis R. Automatic nasal continuous positive airway pressure titration in the laboratory: patient outcomes. Thorax 1997; 52: 72-75.

15. Meurice JC, Marc I, Series F. Efficacy of auto-CPAP in the treatment of obstructive sleep apnea/hypopnea syndrome. Am J Respir Crit Care Med 1996; 153: 794-798.

16. Rechtschaffen A, Kales A. A Manual of Standardized Terminology, Techniques and Scoring System for Sleep Stages of Human Subjects. NIH publication No. 204. Bethesda, MD, National Institutes of Health, 1968.

17. American Sleep Disorders Association. EEG arousals: scoring rules and examples: a preliminary report from the Sleep Disorders Atlas Task Force of the American Sleep Disorders Association. Sleep 1992; 15: 173-184.

18. Young T, Palta M, Dempsey J, Skatrud J, Weber S, Badr S. The occurrence of sleep-disordered breathing among middleaged adults. N Engl J Med 1993; 328: 1230-1235.
19. Berthon-Jones M, Lawrence S, Sullivan CE, Grunstein R Nasal continuous positive airway treatment: current realities and future. Sleep 1996; 19: Suppl. 9, S131-S135.

20. Sharma S, Wall S, Poullot Z, Peters M, Neufeld H, Kryger M. Treatment of obstructive sleep apnea with a self-titrating continuous positive airway pressure (CPAP) system. Sleep 1996; 19: 497-501.

21. Behbehani K, Yen F, Lucas E, Burk J. A sleep laboratory evaluation of an automatic positive airway pressure system for treatment of obstructive sleep apnea. Sleep 1998; 21: 485-491.

22. Berthon Jones M. Feasibility of a self-setting CPAP machine. Sleep 1993; 16: 120-123.

23. Fleetham J, Burt H, Anderson B. Assessment of the efficacy of automatic nasal CPAP therapy in the treatment of obstructive sleep apnea. Am J Respir Crit Care Med 1996; A872.

24. Montserrat JM, Lloberes P, Ballester E, et al. Comparison of manual and automatic CPAP titration in patients with sleep apnea/hypopnea syndrome (SAHS). Am J Respir Crit Care Med 1996; A872.

25. Robert D, Bourdon G, Langevin B, Leger P, Guez A Usefulness and efficacy of an auto adjustable CPAP machine in treatment of OSAS. Am Rev Respir Dis 1993; 147: A680.

26. Series F. Auto CPAP in the treatment of sleep apnea hypopnea syndrome. Sleep 1996; 19: 281-283.

27. Scharf MB, Brannen DE, McDannold MD, Berkowitz DV. Computerized adjustable versus fixed NCPAP treatment of obstructive sleep apnea. Sleep 1996; 19: 491-496.

28. Mathur R, Douglas NJ. Frequency of EEG arousal from nocturnal sleep in normal subjects. Sleep 1995; 18: 330-333. 